\title{
A future for medical genetics: lessons from Catch 22
}

\section{INTRODUCTION}

To paraphrase Milo Minderbinder, the immortal character in Joseph Heller's novel Catch 22, "I lose money on every sale; it's the volume that keeps me in business." There's a lesson for our field in this seemingly twisted logic. Medical Genetics programs are not major sources of revenue for academic medical centers. On the other hand, neither are other intellectually vibrant sectors of the modern medical landscape-including Pediatrics, General Internal Medicine and Psychiatry. Those fields are in no danger of extinction because they are seen by all as necessary for the care of large numbers of patients. Thus, despite the fact that in the surreal world of U.S. medical economics they don't generate much revenue, they persist. It's the volume that keeps them in business.

If we, as medical geneticists, demonstrate that our activities are necessary to patient care, the volume of patients we see will grow and we will emerge as part of medicine's mainstream. If we cannot demonstrate broad usefulness we will not (and don't deserve to) grow beyond the relatively small specialty that we now represent. Thus lies the way forward for Medical Genetics; it is up to us to demonstrate our worth.

From sequence analysis to microarrays, unprecedented amounts of medical information are being generated which will soon directly pertain to patient care. Thus, in this sense too, volume (of information) is a key to our future. Indeed, it is an appealing irony that a major barrier to the quick adoption of genetic information-complexity—may be our most potent ally.

Since the time of Hippocrates, the history of medicine has been one of ever more sophisticated phenotyping: these are the signs of diabetes; those, the symptoms of Alzheimer disease. Medicine's ability to understand and treat disease has hinged on this careful phenotyping of patients. Modern genetics now has an historic opportunity to complete the symmetry of this equation by bringing genotyping to the traditionally phenotypic endeavor of clinical medicine. And while the complexity of this information is a barrier to its implementation, medical geneticists and genetic counselors are well positioned to deal with this emerging volume of information, ensuring our relevance to medical practice. Sources of complexity that thwart the easy use of genetics by clinicians who do not have expertise in our field include the following:

- Variants of uncertain significance (VUS). As more mutational analysis and genotyping is done in medicine, a flood of VUSs will be forthcoming. These are vexing results to interpret and explain to patients. As such they represent a major impediment to broadly based genetic testing in clinical medicine. By the same token, though, the inherent complexity in interpreting genomic data represents a major opportunity for the genetics community since this is precisely our realm of expertise.

- High throughput sequencing applied to the clinic. The barrier to mutation identification in genetically heterogeneous disorders is rapidly disappearing with the advent of highly robust sequencing technologies. Soon the ability to sequence all of the genes involved in disorders such as cardiomyopathy, long QT syndrome, and retinitis pigmentosa will be at hand. Whether the $\$ 1,000$ genome materializes in the next decade or not, there will likely be vast amounts of complex genotypic information increasingly generated in clinical settings.

- Pharmacogenomics. This rapidly emerging area represents the "low hanging fruit" of the genetics revolution. Already (even before actual clinical outcome data are available) the FDA is highlighting the advisability of genotypic guidance in warfarin dosing, a drug that is prescribed more than 20 million times each year in this country. It is the rare cardiologist or generalist who has a firm understanding of how to apply (much less generate) pharmacogenomic data for clinical use. Just as generalists are eager for help in managing diabetes in an increasingly complex environment, we are well-positioned to benefit from a similar trend as genetics increasingly informs therapy throughout medicine.

- Rapidly emerging technologies. The technology used to acquire genetic information is changing rapidly. Different modalities each have their own advantages and disadvantages; proper interpretation of patient test results hinges on both a clinical and technical understanding of the strengths, limitations, and appropriate use of each. We are well-positioned to be central components in this process.

- The emergence of complex databases to collect genotype/ phenotype information. The accession and interpretation of such databases for patient care is daunting to the uninitiated. One can only imagine the challenges that will emerge for the typical nongeneticist seeking to make sense of burgeoning genetic information as it applies to ophthalmology, cardiology, etc.

- Ethical, legal, and social issues (ELSI). For better or for worse, the public accords genetic information a privileged place. The availability of vast amounts of genotypic data will accentuate old problems and generate new ELSI issues which have long been the bread and butter of practicing geneticists.

We must serve as interpreters of increasingly complex genetics information in the clinical setting and teach others how it can be applied to the benefit of their patients. If we fail to show that our discipline embodies general principles 
and concepts that will benefit the emergence of personalized medicine and clinical research, the various specialties will each go it alone, but in a slower and less effective manner.

An example of the recent emergence of modern genetics into the clinical arena is in the practice of oncology. Genetic assessment has recently become an integral part of the evaluation and care of patients with breast and ovarian cancers. While oncologists at many institutions have, to their credit, stepped up to the plate, I would submit that given our backgrounds, training and orientation, our involvement can implement the proper use of genetics in medicine more quickly and more completely than that accomplished by the nongenetics specialist alone.

All of medicine rests on effective teamwork and the task before us is no exception. We can act as midwives to bring genetics to the rest of clinical medicine only by partnering with others who are hungry for its application. In that vein we must establish formal partnerships; a few suggestions, many of which are being implemented at my institution, follow.

- Collaborative Clinics. Nongeneticist clinicians typically know little about our field and are unsure of what we have to offer. Thus, we must seek them out to partner with, using the established model that has been successful at many institutions in the realm of cancer genetics. One avenue by which to accomplish this is to establish collaborative clinics in which a counselor and/or geneticist sees patients with the specialists in their clinic and begins to apply genetic testing and counseling when appropriate. By designing such clinics around those diseases that are most susceptible to the application of emerging genetic technologies we can quickly demonstrate our worth. These clinics can start small. Once each month the counselor or geneticist can join the nongenetics clinician and begin the process of becoming a part of the care of their patients. Our experience is that most nongenetics clinicians are eager to partner with us, and we have established such clinics in cooperation with cardiology, ophthalmology, dermatology, nephrology, oncology, and hematology (with regard to thrombosis and hemostasis).

- The laboratory. We are a necessary bridge spanning the clinical pathology lab and the clinic. As more (and more complex) genotypic data are routinely generated, the laboratory will need help interpreting VUS and deciding on the most appropriate genetic analyses to perform. Moreover, the probabilistic interpretation of many genetic testing results cannot be adequately conveyed in a simple lab report in the same way that a hematocrit can. We are the link between the lab and the end users of this technology, able to triangulate among the lab, the clinician, and the patient.

- Novel clinics and consult services. The identification of clinical needs must be followed quickly by easy mechanisms to bring genetics into the mainstream. An obvious candidate is a pharmacogenomic clinic/consult service. Most institutions already have a warfarin clinic that manages innumerable patients who need to be on this dangerous agent. Thus an infrastructure already exists and genetics can be readily integrated into such clinics to quickly deploy our emerging knowledge of pharmacogenetics in this clinical situation.

- Genetic counselors. We can't accomplish the integration of genetics into medicine by ourselves; genetic counselors are our natural allies and we are theirs. Of course we must ensure appropriate supervision predicated upon the broad understanding of medicine that MDs have by virtue of their training. However genetic counselors are professionals in their own right and deserve a significant degree of autonomy. I see little evidence to suggest that genetic counselors are intent on usurping our role and "practicing medicine;" indeed, I can think of few professions that are more team-oriented than that of the counseling community. Many already interface with a variety of different specialists. It is not only MD geneticists who can act in a supervisory role to counselors, as the OB/GYN and oncology communities have well demonstrated. In the end, from a purely selfish standpoint, it is imperative to our own future that we ensure the viability and sustainability of genetic counseling as a profession. We need to set ourselves the goal of working hand-in-hand with the counseling community to help genetic counselors obtain licensure so that they can charge for their services, thus ensuring a stable future for their profession and for ours.

To overcome the obvious economic realities that face our field, a gradual approach is appropriate. At UNC, we have been deploying clinician and counselor staff to collaborative clinics in a modest fashion on a monthly basis. As we increasingly demonstrate our usefulness and become an expected and necessary part of the clinical landscape, we will establish remuneration in the form of contracts with the appropriate partnering clinics. Other institutions may use different models to put genetic services on a firm financial footing.

Finally, we often lament the paucity of young physicians who choose to pursue subspecialty training in our field. How can this be when, here at the outset of the $21^{\text {st }}$ century, genetics easily stands as one of the most intellectually exciting arenas of human thought - not to mention one of the most fascinating fields of medicine? I think that the answer lies in the fact that most physicians are not driven exclusively by intellectual curiosity. Academic geneticists aside, physicians are generally an intensely practical breed, leavened, hopefully with idealism. At the end of the day, to satisfy both their practicality and idealism they want to know what they can do to help their patients. Thus, intellectual excitement alone will not solve the lack of genetics trainees. Our ranks will swell only when we demonstrate our usefulness. Once we do so, students will be interested in 
joining us and our trainee deficit will, with appropriate education and publicity, take care of itself.

Medical genetics has the ability to lead the way into a new era of medicine. To do so- and at the same time ensure our own future-we must, above all, demonstrate our usefulness by reaching out to the physicians and patients who can benefit from our knowledge. We have much to offer the rest of medicine. It would be a tragedy for our patients, as well as a sad irony, if medical genetics sits out the "genetic revolution."

James P. Evans, MD, PhD

University of North Carolina, Department of Genetics 